\title{
A N ew Group-Contribution Method for the Estimation of Physical Properties of Hydrocarbons
}

\author{
N. Skander and C.E. Chitour ${ }^{2}$ \\ 1 Centre de Recherche et développement de Sonatrach, avenue du $1^{\text {er }} \mathrm{N}$ ovembre, 35000 Boumerdès - Algérie \\ 2 Laboratoire de Valorisation des énergies fossiles, Département de génie chimique, \\ École nationale polytechnique, Alger - Algérie \\ e-mail: lavalef@yahoo.fr
}

\begin{abstract}
Résumé - Nouvelle méthode de contribution de groupes pour l'estimation des propriétés physiques des hydrocarbure - Dans le présent article, nous proposons une nouvelle méthode de contribution de groupes pour l'estimation de la température d'ébullition, du point de congélation et de la densité liquide à $20^{\circ} \mathrm{C}$ des hydrocarbures purs. La méthode a été établie avec une assez bonne précision et, comparée à d'autres méthodes de contributions de groupes, elle a enregistré de meilleurs résultats en termes de déviations standard moyennes.
\end{abstract}

Abstract-A New Group-Contribution Method for the Estimation of Physical Properties of Hydrocarbons - In the present work, we propose a new group-contribution method to estimate the boiling point, freezing point and the liquid density at $20^{\circ} \mathrm{C}$ of hydrocarbons. The method was tested with satisfactory accuracy, and when compared with other group-contribution methods, it reveals better results in terms of average standard deviations.

\section{NOTATIONS}

$a, b, c, d, m, n, p \quad$ constants for each property in Equation $\mathrm{F}$

$d_{20}$ liquid density at $20^{\circ} \mathrm{C}\left(\mathrm{kg} / \mathrm{m}^{3}\right)$

$F \quad$ mathematical function

$M \quad$ molecular weight $(\mathrm{g} / \mathrm{mol})$

$T_{b} \quad$ normal boiling point (K)

$T_{F} \quad$ freezing point (K).

\section{Greek letters}

$\theta \quad$ a given property

$\Delta \theta_{i}$ contribution of the group $i$ for the evaluation of the property $\theta$.

\section{IN TRO DUCTION}

Physical properties of hydrocarbons are required in the design of most petroleum processing equipments. These characteristics can be obtained through experimental measurements, graphical methods or analytical equations. The most widely used are those of group-contribution methods such those of Lydersen (1955), Joback (1984), Ambrose (1980), Klincewicz and Reid (1984), Constantinou et al. (1994), Constantinou et al. (1995), which are based only on the molecular structure of the compound. The property is estimated by a summation of the contributions of individual groups and fragments which constitute the molecule. Even if these correlations are able to estimate the 
properties quite rapidly, many of them fail in distinguishing among isomers due to the oversimplification of the molecule structure or, in extrapolating to heavier compounds.

In this work, we have proposed a group-contribution method which allows to capture isomers differences to estimate boiling point $(\mathrm{K})$, freezing point $(\mathrm{K})$ and liquid density at $20^{\circ} \mathrm{C}\left(\mathrm{kg} / \mathrm{m}^{3}\right)$ of pure hydrocarbons. The average absolute deviations of the new correlations are $0.77 \%, 8.44 \%$ and $1.30 \%$ respectively for boiling point, freezing point and density.

\section{PROPOSED METHOD}

This work was carried out on the basis of a compiled properties values of pure compounds, obtained exclusively from the TRC data bank (TRC, Thermodynamic Data Base, version $1.3,1994)$. The study concerns only hydrocarbons belonging to the following families: $n$-paraffins, isoparaffins, olefins, alcyns, naphthenes and aromatics, because of their predominance in petroleum industry. Condensed rings are not considered in this work, an extension of the present work to theses types of molecules will be done in the future.

On the basis of the molecule's structures of the data bank compounds, we selected first 10 types of groups: $\mathrm{CH}_{3}, \mathrm{CH}_{2}$, $\mathrm{CH}, \mathrm{C},=\mathrm{CH}_{2},=\mathrm{CH},=\mathrm{C},=\mathrm{C}=$, $\equiv \mathrm{CH}$ and $\equiv \mathrm{C}$, without any distinction between aliphatic and ring chains. Therefore, we admit at first sight that each group has a single contribution when appearing in a paraffinic, naphthenic or aromatic molecule. Then, a set of 42 other groups was added to distinguish between the different families and also between isomers whether in aliphatic or ring structures. In developing these correction groups, we take into account the proximity effects of the $\mathrm{CH}_{3}$ groups. In the same way, we affect correction terms to saturated rings in naphthenes and to the benzene ring, in addition to those of the position cis- and trans- in olefinic and naphthenic molecules, and ortho, meta, para and other tri- and tetra-substituted positions.

After generating the group additivity parameters, we proceed to the analysis of the data from the experimental values of boiling point $(\mathrm{K})$, freezing point $(\mathrm{K})$ and liquid density $\left(\mathrm{kg} / \mathrm{m}^{3}\right)$.

To develop a group additivity relationship, we try several equations which can be generalised in mathematical form by the following relationship:

$$
\mathrm{F}(\theta)=a+b \Sigma \Delta \theta_{i}+c\left(\Sigma \Delta \theta_{i}\right)^{m}+d\left(\Sigma \Delta \theta_{i}\right)^{n}
$$

where $\theta$ is a given property and $\mathrm{F}(\theta)$ a function equal to $\theta$, $\exp (\theta / p), 1 / \theta^{\mathrm{p}}, \mathrm{M} / \theta$ or $T_{b} / \theta$. The parameters $a, b, c, d, m, n$ and $p$ are constants determined by the regression. $T_{b}(\mathrm{~K})$ and $M(\mathrm{~g} / \mathrm{mol})$ are respectively the boiling point and the molecular weight.
Twenty equations can be derived from the generalised equation according to the shape of the function $\mathrm{F}$ and by cancelling one or several parameters in the expression of the generalised correlation, like shown in Table 1. The aim of this approach is to test different forms of mathematical equations to provide the best correlation with the minimum error.

TABLE 1

The different forms of Equation $\mathrm{F}(\theta)$

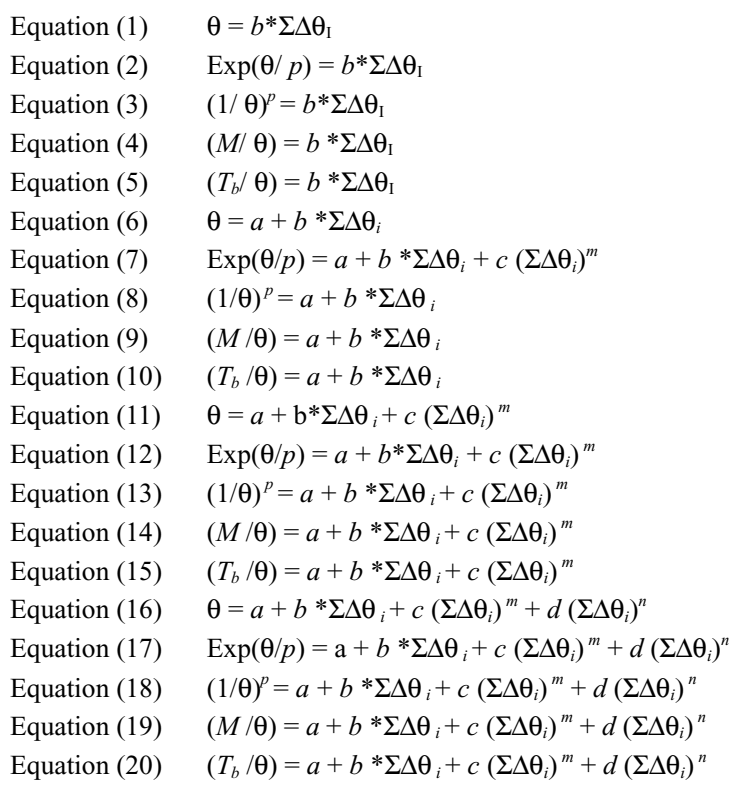

The nonlinear regression of the data was conducted using the Levenberg-Marquardt algorithm for each property. In order to test the reliability of the different equations, we determine for each one the average absolute deviations AAD (\%) registered in the case of each hydrocarbon family.

The results obtained are summarised in Tables 2, 3 and 4 respectively for boiling point, freezing point and density. The number of data used for each regression, and their distribution in terms of carbon atom numbers are also given in these tables.

In selecting the best equation that best fitted the data, we compare between the deviations registered by the different equations tested taking into account all the families. Also, we compare their ability in extrapolating the data to heavier compounds.

\subsection{Selecting Equation for Estimating Boiling Point}

For boiling point, we registered the higher deviations for the first terms of each family, particularly for ones that are in the gaseous state at ambient temperature. Equations for which we didn't give any results, have been eliminated because 
they provided some very important gaps in relation to reference data.

The results given in Table 2 show that Equations (11, 14, 16, 17 and 19) give the smallest average deviations. In a first approach, we select them as the best equations for the evaluation of the boiling point of pure hydrocarbons. In a second stage, we compare their ability to predict this property for hydrocarbons with high carbon numbers. For this purpose, we take like reference the Riazi and El Sahhaf (1995) Equation that joins the boiling points of normalparaffins to their carbon atom numbers. Indeed, the application of this correlation to heavy compounds has been largely proved. On this basis, we select Equation (11) as the best correlation that satisfies the two criteria of selection.

\subsection{Selecting Equation for Estimating Freezing Point}

In the case of the freezing point, like shown in Table 3 , the deviations obtained comparatively to the reference data remain high enough for most hydrocarbons, with the exception of normal paraffins. In spite of this, we kept equations that provided the relatively weakest errors. So, we selected Equations (4, 5, 9, 14 and 16) in a first step. Thereafter, and while comparing their faculty to estimate correctly freezing points of heavy $n$-paraffins while taking in reference the correlation of Riazi and El Sahhaf (1995), we keep the Equation (14) as the group-contribution correlation which predict best this property.

\subsection{Selecting Equation for Estimating Liquid Density}

For the density, results given in Table 4 show that the registered deviations are in general satisfactory, with the exception of the aromatics that sometimes give some elevated errors, especially for the first terms of this hydrocarbon family. While comparing the average deviations registered on the set of hydrocarbons, Equations (2, 4, 7, 9, $14,15,19$ and 20) are selected as those that provided the best results. As far as the second criteria of selection is concerned, we take as reference the value of $856 \mathrm{~kg} / \mathrm{m}^{3}$ given by Wuithier (1972) as the limiting value toward which tend the liquid density of long chain hydrocarbons. As a result, Equation (9) is kept for the prediction of this property.

\subsection{Equations Parameters}

The values of the group-contribution parameters and those of the constants $a, b, c, d, m, n$ and $p$ of the selected equations are given in Table 5 .

TABLE 2

Comparison between the AAD of the different equations predicting boiling point

\begin{tabular}{|c|c|c|c|c|c|c|c|}
\hline & $n$-paraffins & $i$-paraffins & Olefins & Alcyns & Naphthenes & Aromatics & $\operatorname{AAD}(\%)$ \\
\hline Data points & 79 & 761 & 293 & 87 & 327 & 256 & 1803 \\
\hline Carbon range & $\mathrm{C}_{2}-\mathrm{C}_{80}$ & $\mathrm{C}_{4}-\mathrm{C}_{100}$ & $\mathrm{C}_{2}-\mathrm{C}_{100}$ & $\mathrm{C}_{2}-\mathrm{C}_{40}$ & $\mathrm{C}_{3}-\mathrm{C}_{102}$ & $\mathrm{C}_{6}-\mathrm{C}_{102}$ & \\
\hline Eq. (1) & - & - & - & - & - & - & - \\
\hline Eq. (2) & 1.10 & 0.97 & 1.36 & 1.73 & 1.60 & 1.52 & 1.27 \\
\hline Eq. (3) & 2.83 & 1.24 & 2.93 & 3.09 & 4.77 & 2.73 & 2.53 \\
\hline Eq. (4) & - & - & - & - & - & - & - \\
\hline Eq. (6) & - & - & - & - & - & - & - \\
\hline Eq. (7) & 1.10 & 0.97 & 1.36 & 1.73 & 1.60 & 1.52 & 1.27 \\
\hline Eq. (8) & 2.83 & 1.24 & 2.92 & 3.09 & 4.75 & 2.74 & 2.52 \\
\hline Eq. (9) & 2.02 & 1.17 & 2.35 & 4.24 & 2.07 & 1.77 & 1.80 \\
\hline Eq. (11) & 0.11 & 0.78 & 0.61 & 0.39 & 0.99 & 0.95 & 0.77 \\
\hline Eq. (12) & 0.64 & 0.99 & 1.01 & 1.44 & 1.15 & 1.11 & 1.05 \\
\hline Eq. (13) & 2.83 & 1.24 & 2.93 & 3.09 & 4.75 & 2.73 & 2.52 \\
\hline Eq. (14) & 0.15 & 0.74 & 0.74 & 0.42 & 0.96 & 0.88 & 0.76 \\
\hline Eq. (16) & 0.12 & 0.78 & 0.61 & 0.39 & 1.00 & 0.89 & 0.76 \\
\hline Eq. (17) & 0.37 & 0.88 & 0.72 & 1.20 & 1.04 & 0.96 & 0.89 \\
\hline Eq. (18) & 2.83 & 1.24 & 2.92 & 3.09 & 8.69 & 2.74 & 3.24 \\
\hline Eq. (19) & 0.15 & 0.74 & 0.72 & 0.42 & 0.91 & 0.87 & 0.74 \\
\hline
\end{tabular}

* Equations (5, 10, 15 and 20) are not considered for the boiling point. 
TABLE 3

Comparison between the $\mathrm{AAD}$ of the different equations predicting freezing point

\begin{tabular}{c|c|c|c|c|c|c|c}
\hline & $\boldsymbol{n}$-paraffins & $\boldsymbol{i}$-paraffins & Olefins & Alcyns & Naphthenes & Aromatics & AAD (\%) \\
\hline Data points & $\mathbf{3 9}$ & $\mathbf{8 8}$ & $\mathbf{1 2 1}$ & $\mathbf{5 4}$ & $\mathbf{1 5 3}$ & $\mathbf{1 2 2}$ & $\mathbf{5 7 7}$ \\
\hline Carbon range & $\mathbf{C}_{2}$ - $\mathbf{C}_{40}$ & $\mathbf{C}_{\mathbf{4}}-\mathbf{C}_{20}$ & $\mathbf{C}_{2}-\mathbf{C}_{\mathbf{4 0}}$ & $\mathbf{C}_{2}-\mathbf{C}_{40}$ & $\mathbf{C}_{\mathbf{3}} \mathbf{C}_{42}$ & $\mathbf{C}_{\mathbf{6}}-\mathbf{C}_{\mathbf{4 2}}$ & - \\
\hline Eq. (1) & - & - & - & - & - & - & - \\
Eq. (2) & 4.20 & 9.81 & 10.65 & 8.12 & 11.11 & 8.76 & $\mathbf{9 . 5 7}$ \\
Eq. (3) & - & - & - & - & - & - & - \\
Eq. (4) & 2.81 & 9.32 & 8.35 & 8.08 & 9.18 & 9.17 & $\mathbf{8 . 4 9}$ \\
Eq. (5) & 3.51 & 10.51 & 9.31 & 6.09 & 9.43 & 9.76 & $\mathbf{8 . 9 3}$ \\
Eq. (6) & - & - & - & - & - & - & - \\
Eq. (7) & 4.20 & 9.81 & 10.64 & 8.12 & 11.09 & 8.76 & $\mathbf{9 . 5 6}$ \\
Eq. (8) & - & - & - & - & - & - & - \\
Eq. (9) & 2.81 & 9.32 & 8.35 & 8.09 & 4.30 & 9.17 & $\mathbf{7 . 2 0}$ \\
Eq. (10) & 3.51 & 10.51 & 9.31 & 6.10 & 9.44 & 9.77 & $\mathbf{8 . 9 3}$ \\
Eq. (11) & 2.42 & 9.71 & 9.47 & 8.09 & 9.98 & 9.15 & $\mathbf{8 . 9 7}$ \\
Eq. (12) & 2.33 & 9.8 & 9.64 & 8.21 & 10.01 & 9.16 & $\mathbf{9 . 0 3}$ \\
Eq. (13) & 5.78 & 9.50 & 11.81 & 8.17 & 12.67 & 8.55 & $\mathbf{1 0 . 2 5}$ \\
Eq. (14) & 1.94 & 9.37 & 8.25 & 8.34 & 9.40 & 8.87 & $\mathbf{8 . 4 4}$ \\
Eq. (15) & 2.03 & 10.78 & 11.21 & 7.45 & 13.74 & 10.80 & $\mathbf{1 0 . 7 6}$ \\
Eq. (16) & 2.38 & 9.74 & 9.50 & 8.13 & 9.96 & 9.15 & $\mathbf{8 . 9 8}$ \\
Eq. (17) & 1.16 & 12.11 & 10.79 & 7.81 & 12.19 & 9.27 & $\mathbf{1 0 . 1 1}$ \\
Eq. (18) & 2.33 & 9.78 & 9.55 & 8.21 & 13.92 & 9.19 & $\mathbf{1 0 . 0 5}$ \\
Eq. (19) & 1.94 & 9.37 & 8.29 & 8.31 & 9.49 & 12.16 & $\mathbf{9 . 1 6}$ \\
Eq. (20) & 1.78 & 10.23 & 9.98 & 7.74 & 11.41 & 10.63 & $\mathbf{9 . 7 7}$ \\
\hline
\end{tabular}

TABLE 4

Comparison between the $\mathrm{AAD}$ of the different equations predicting liquid density at $20^{\circ} \mathrm{C}$

\begin{tabular}{c|c|c|c|c|c|c|c}
\hline & $\boldsymbol{n}$-paraffins & $\boldsymbol{i}$-paraffins & Olefins & Alcyns & Naphthenes & Aromatics & AAD (\%) \\
\hline Data points & $\mathbf{3 5}$ & $\mathbf{6 7 7}$ & $\mathbf{2 2 7}$ & $\mathbf{8 5}$ & $\mathbf{2 6 4}$ & $\mathbf{1 9 7}$ & $\mathbf{1 4 8 5}$ \\
\hline Carbon range & $\mathbf{C}_{5}-\mathbf{C}_{40}$ & $\mathbf{C}_{5}-\mathbf{C}_{20}$ & $\mathbf{C}_{4}-\mathbf{C}_{40}$ & $\mathbf{C}_{4}-\mathbf{C}_{40}$ & $\mathbf{C}_{4}-\mathbf{C}_{42}$ & $\mathbf{C}_{6}-\mathbf{C}_{40}$ & - \\
\hline Eq. (1) & 2.37 & 1.21 & 2.23 & 2.41 & 3.09 & 3.39 & $\mathbf{2 . 0 9}$ \\
Eq. (2) & 0.46 & 1.11 & 1.30 & 1.66 & 1.95 & 3.11 & $\mathbf{1 . 5 7}$ \\
Eq. (3) & - & - & - & - & - & - & - \\
Eq. (4) & 0.19 & 0.89 & 0.93 & 0.92 & 2.76 & 1.57 & $\mathbf{1 . 3 0}$ \\
Eq. (5) & - & - & - & - & - & - & - \\
Eq. (6) & 2.37 & 1.21 & 2.23 & 2.41 & 3.09 & 3.55 & $\mathbf{2 . 1 1}$ \\
Eq. (7) & 0.46 & 1.11 & 1.30 & 1.66 & 1.95 & 3.12 & $\mathbf{1 . 5 7}$ \\
Eq. (8) & - & - & - & - & - & - & - \\
Eq. (9) & 0.19 & 0.89 & 0.93 & 0.92 & 2.76 & 1.57 & $\mathbf{1 . 3 0}$ \\
Eq. (10) & - & - & - & - & - & - & - \\
Eq. (11) & 0.15 & 1.03 & 1.15 & 1.51 & 2.54 & 9.11 & $\mathbf{2 . 4 0}$ \\
Eq. (12) & 0.10 & 1.04 & 1.15 & 1.47 & 2.44 & 8.73 & $\mathbf{2 . 3 3}$ \\
Eq. (13) & 0.15 & 1.03 & 1.15 & 1.51 & 2.53 & 9.32 & $\mathbf{2 . 4 2}$ \\
Eq. (14) & 0.09 & 0.89 & 0.91 & 0.94 & 2.67 & 1.57 & $\mathbf{1 . 2 8}$ \\
Eq. (15) & 0.09 & 0.56 & 0.61 & 0.69 & 1.97 & 1.11 & $\mathbf{0 . 8 9}$ \\
Eq. (16) & 0.13 & 1.04 & 1.15 & 1.51 & 2.01 & 9.25 & $\mathbf{2 . 3 2}$ \\
Eq. (17) & 0.08 & 1.04 & 1.15 & 1.50 & 2.50 & 8.60 & $\mathbf{2 . 3 2}$ \\
Eq. (18) & 0.12 & 1.04 & 1.15 & 1.50 & 2.48 & 8.88 & $\mathbf{2 . 3 6}$ \\
Eq. (19) & 0.06 & 0.90 & 0.94 & 0.98 & 2.17 & 1.59 & $\mathbf{1 . 2 1}$ \\
Eq. (20) & 0.10 & 0.56 & 0.62 & 0.70 & 1.97 & 1.11 & $\mathbf{0 . 8 9}$ \\
\hline
\end{tabular}


TABLE 5

Parameters values and group increments values of Equation $\mathrm{F}$

\begin{tabular}{|c|c|c|c|}
\hline & $T_{b}$ & $T_{F}$ & $d_{20}$ \\
\hline Equation & Eq. (11) & Eq. (14) & Eq. (9) \\
\hline \multicolumn{4}{|l|}{ Parameter values } \\
\hline$p$ & - & - & - \\
\hline$a$ & $2.10497 \mathrm{E}+03$ & $-5.32928 \mathrm{E}-01$ & $-3.91046 \mathrm{E}-01$ \\
\hline$b$ & $-1.77440 \mathrm{E}-01$ & $3.05306 \mathrm{E}-01$ & $3.49011 \mathrm{E}-01$ \\
\hline$c$ & $-6.19434 \mathrm{E}+03$ & $6.06110 \mathrm{E}-01$ & - \\
\hline$d$ & - & - & - \\
\hline$m$ & $-2.80170 \mathrm{E}-01$ & $-4.38977 \mathrm{E}-01$ & - \\
\hline$n$ & - & - & - \\
\hline \multicolumn{4}{|l|}{ Contributions } \\
\hline$-\mathrm{CH}_{3}$ & $3.36103 \mathrm{E}+01$ & $6.80429 \mathrm{E}-01$ & $6.53292 \mathrm{E}-01$ \\
\hline$-\mathrm{CH}_{2-}$ & 6.01945 & $1.23893 \mathrm{E}-01$ & $4.67606 \mathrm{E}-02$ \\
\hline$-\mathrm{CH}<$ & $-2.28076 \mathrm{E}+01$ & $-4.92776 \mathrm{E}-01$ & $-5.76890 \mathrm{E}-01$ \\
\hline$>\mathrm{C}<$ & $-5.10314 \mathrm{E}+01$ & -1.30057 & -1.20711 \\
\hline$=\mathrm{CH}_{2}$ & $3.29791 \mathrm{E}+01$ & $6.61299 \mathrm{E}-01$ & $6.52976 \mathrm{E}-01$ \\
\hline$=\mathrm{CH}_{-}^{-}$ & 6.33765 & $2.93538 \mathrm{E}-01$ & $3.46579 \mathrm{E}-02$ \\
\hline$=\mathrm{C}<$ & $-2.11492 \mathrm{E}+01$ & $-6.60729 \mathrm{E}-01$ & $-5.89958 \mathrm{E}-01$ \\
\hline$=\mathrm{C}=$ & 7.94780 & $-2.35470 \mathrm{E}-01$ & $2.10506 \mathrm{E}-02$ \\
\hline$\equiv \mathrm{CH}$ & $3.27471 \mathrm{E}+01$ & $6.15015 \mathrm{E}-01$ & $6.39500 \mathrm{E}-01$ \\
\hline$\equiv \mathrm{C}_{-}$ & 7.19358 & $9.32368 \mathrm{E}-02$ & $2.28269 \mathrm{E}-02$ \\
\hline \multicolumn{4}{|c|}{ Corrections terms owed to the $\mathrm{CH}_{3}$ proximity effects } \\
\hline $\mathrm{C}\left(\mathrm{CH}_{3}\right)_{3}$ & -1.73388 & $3.87264 \mathrm{E}-01$ & $4.01010 \mathrm{E}-02$ \\
\hline $\mathrm{C}\left(\mathrm{CH}_{3}\right)_{2}$ & $-9.13930 \mathrm{E}-01$ & $3.57568 \mathrm{E}-01$ & $1.99574 \mathrm{E}-02$ \\
\hline $\mathrm{C}\left(\mathrm{CH}_{3}\right)$ & $-5.50980 \mathrm{E}-01$ & $2.64643 \mathrm{E}-01$ & 4.70667E-03 \\
\hline $\mathrm{C}\left(\mathrm{CH}_{3}\right) \mathrm{C}\left(\mathrm{CH}_{3}\right)$ & $7.10180 \mathrm{E}-01$ & $-1.52738 \mathrm{E}-01$ & $2.08435 \mathrm{E}-03$ \\
\hline $\mathrm{C}\left(\mathrm{CH}_{3}\right) \mathrm{C}\left(\mathrm{CH}_{3}\right)_{2}$ & 1.21475 & 7.38494E-02 & $1.44740 \mathrm{E}-04$ \\
\hline $\mathrm{C}\left(\mathrm{CH}_{3}\right) \mathrm{C}\left(\mathrm{CH}_{3}\right)_{3}$ & 1.82757 & $2.48059 \mathrm{E}-03$ & $-5.22518 \mathrm{E}-03$ \\
\hline $\mathrm{C}\left(\mathrm{CH}_{3}\right)_{2} \mathrm{C}\left(\mathrm{CH}_{3}\right)_{2}$ & 2.12237 & $9.90933 \mathrm{E}-02$ & $-4.61323 \mathrm{E}-03$ \\
\hline $\mathrm{C}\left(\mathrm{CH}_{3}\right)_{2} \mathrm{C}\left(\mathrm{CH}_{3}\right)_{3}$ & 3.45589 & $-6.34782 \mathrm{E}-01$ & $-1.03234 \mathrm{E}-02$ \\
\hline \multicolumn{4}{|c|}{ Corrections terms owed to the types of positions } \\
\hline cis- & $-1.42400 \mathrm{E}-01$ & $7.82603 \mathrm{E}-02$ & $2.37399 \mathrm{E}-03$ \\
\hline trans- & $-2.01200 \mathrm{E}-01$ & $-6.96987 \mathrm{E}-02$ & 4.04111E-03 \\
\hline \multicolumn{4}{|l|}{ Corrections terms owed to the ring structure } \\
\hline 3 membered ring & $5.67167 \mathrm{E}+01$ & $-6.70642 \mathrm{E}-02$ & 1.21925 \\
\hline 4 membered ring & $5.67215 \mathrm{E}+01$ & 1.14841 & 1.19909 \\
\hline 5 membered ring & $5.67392 \mathrm{E}+01$ & 1.63831 & 1.20410 \\
\hline 6 membered ring & $5.79497 \mathrm{E}+01$ & 1.31182 & 1.18562 \\
\hline 7 membered ring & $5.99484 \mathrm{E}+01$ & $5.96939 \mathrm{E}-02$ & 1.14001 \\
\hline 8 membered ring & $6.15173 \mathrm{E}+01$ & $1.75399 \mathrm{E}-01$ & 1.13091 \\
\hline 9 membered ring & $6.24661 \mathrm{E}+01$ & $4.60262 \mathrm{E}-01$ & 1.12504 \\
\hline 10 membered ring & $6.29563 \mathrm{E}+01$ & $6.05954 \mathrm{E}-01$ & 1.12153 \\
\hline 11 membered ring & $6.25620 \mathrm{E}+01$ & 1.04145 & 1.11894 \\
\hline 12 membered ring & $6.21962 \mathrm{E}+01$ & $4.11652 \mathrm{E}-01$ & 1.11816 \\
\hline 13 membered ring & $6.18293 \mathrm{E}+01$ & $7.85851 \mathrm{E}-01$ & 1.11867 \\
\hline 14 membered ring & $6.10686 \mathrm{E}+01$ & $5.98616 \mathrm{E}-01$ & 1.11929 \\
\hline 15 membered ring & $6.05805 \mathrm{E}+01$ & $5.91201 \mathrm{E}-01$ & 1.12084 \\
\hline 16 membered ring & $5.99864 \mathrm{E}+01$ & $6.59633 \mathrm{E}-01$ & 1.12262 \\
\hline 17 membered ring & $5.92470 \mathrm{E}+01$ & $6.81649 \mathrm{E}-01$ & 1.12462 \\
\hline 18 membered ring & $5.83214 \mathrm{E}+01$ & $6.68171 \mathrm{E}-01$ & 1.12685 \\
\hline 19 membered ring & $5.76199 \mathrm{E}+01$ & - & 1.12931 \\
\hline 20 membered ring & $5.66837 \mathrm{E}+01$ & - & 1.13200 \\
\hline \multicolumn{4}{|c|}{ Corrections terms owed to the aromatic structures } \\
\hline Aromatic ring & $5.61560 \mathrm{E}+01$ & $4.97120 \mathrm{E}-01$ & 1.20018 \\
\hline Ortho-substitution & $2.87670 \mathrm{E}-01$ & 4.03396E-01 & $5.95223 \mathrm{E}-03$ \\
\hline Meta-substitution & $7.10900 \mathrm{E}-02$ & $5.57575 \mathrm{E}-01$ & $1.15111 \mathrm{E}-02$ \\
\hline Para-substitution & $7.10900 \mathrm{E}-02$ & $5.57575 \mathrm{E}-01$ & $1.15111 \mathrm{E}-02$ \\
\hline Substitution in positions $1-2-3$ & 1.29240 & $5.74670 \mathrm{E}-01$ & $1.24819 \mathrm{E}-02$ \\
\hline Substitution in positions 1-2-4 & $9.73490 \mathrm{E}-01$ & 7.93313E-01 & $2.10110 \mathrm{E}-02$ \\
\hline Substitution in positions $1-3-5$ & $-6.73900 \mathrm{E}-01$ & $9.72197 \mathrm{E}-01$ & $2.43517 \mathrm{E}-02$ \\
\hline Substitution in positions $1-2-5$ & 1.30243 & $4.36259 \mathrm{E}-01$ & $1.33045 \mathrm{E}-02$ \\
\hline Substitution in positions $1-2-6$ & 1.14308 & $4.47632 \mathrm{E}-01$ & $1.13885 \mathrm{E}-02$ \\
\hline Substitution in positions 1-3-4 & $-3.53680 \mathrm{E}-01$ & $9.50403 \mathrm{E}-01$ & $1.95087 \mathrm{E}-02$ \\
\hline Substitution in positions 1-2-4-5 & 2.40917 & $4.63746 \mathrm{E}-01$ & $3.13636 \mathrm{E}-02$ \\
\hline Substitution in positions 1-2-3-4 & 4.09709 & $7.29045 \mathrm{E}-01$ & $2.31769 \mathrm{E}-02$ \\
\hline Substitution in positions $1-2-3-5$ & 2.32485 & $9.25268 \mathrm{E}-01$ & $2.99362 \mathrm{E}-02$ \\
\hline Substitution in positions $1-2-3-4-5$ & 7.39249 & $7.34520 \mathrm{E}-01$ & $3.21448 \mathrm{E}-02$ \\
\hline
\end{tabular}




\section{METHOD ACCURACY}

In order to test the accuracy of the proposed equations, we have compared their average absolute deviations to those registered by other methods given in literature and using the chemical structure to predict the studied properties. The results obtained and given in Table 6 show that our method give in each case the best results with regard to reference data, notably in the case of the boiling point.

\section{EXAMPLES}

To illustrate the proposed method, we provide in Tables 7 to 14 the estimation of the boiling point, freezing point and liquid density of $n$-hexadecane ( $n$-paraffin), 2,3,3-trimethylpentane (iso-paraffin), 2-methyl-1-nonene (olefin), 1-dodecyne (alcyn), 1-ethyl-cis-2-methylcyclopentane (naphthene), 1,1-dicyclohexyl-dodecane (naphthene), 1,4-isopropyltoluene (aromatic) and 2,4'-dimethyldiphenylmethane (aromatic).

TABLE 6

Comparison of the accuracy between existing correlations and the proposed equations

\begin{tabular}{|c|c|c|c|c|c|c|}
\hline & $n$-paraffins & $i$-paraffins & Olefins & Alcyns & Naphthenes & Aromatics \\
\hline \multicolumn{7}{|c|}{ AAD (\%) } \\
\hline Proposed method & 0.11 & 0.78 & 0.61 & 0.39 & 0.99 & 0.95 \\
\hline Joback (1984) & 48.79 & 9.62 & 2.29 & 7.70 & 24.22 & 29.48 \\
\hline Constantinou (1994) & 6.28 & 1.89 & 3.27 & 3.98 & 3.59 & 3.42 \\
\hline Kreglewski-Zwolinsky (1961) & 0.22 & - & - & - & - & - \\
\hline Riazi and El Sahhaf (1995) & 0.22 & - & - & - & - & - \\
\hline \multicolumn{7}{|c|}{$\begin{array}{ll}\text { Freezing point } & \text { AAD }(\%)\end{array}$} \\
\hline Proposed method & 1.94 & 9.37 & 8.25 & 8.34 & 9.40 & 8.87 \\
\hline Joback (1984) & 19.67 & 10.38 & 12.84 & 31.47 & 22.06 & 20.62 \\
\hline Constantinou (1994) & 8.34 & 14.96 & 7.48 & 14.50 & 11.45 & 9.01 \\
\hline Riazi and El Sahhaf (1995) & 2.77 & - & - & - & - & - \\
\hline
\end{tabular}

TABLE 7

Estimation of the boiling point, freezing point and liquid density of $n$-hexadecane

\begin{tabular}{c|c|c|c}
\hline Groups & $\boldsymbol{T}_{\boldsymbol{b}}$ & $\boldsymbol{T}_{\boldsymbol{F}}$ & $\boldsymbol{d}_{\mathbf{2 0}}$ \\
\hline $\begin{array}{c}2-\mathrm{CH}_{3} \\
14-\mathrm{CH}_{2}-\end{array}$ & $2 \times 33.6103$ & $2 \times 0.680429$ & $2 \times 0.653292$ \\
$14 \times 6.01945$ & $14 \times 0.123893$ & $14 \times 0.0467606$ \\
\hline Calculated value & $560.6 \mathrm{~K}$ & $289.9 \mathrm{~K}$ & $771.7 \mathrm{~kg} / \mathrm{m}^{3}$ \\
\hline Experimental value & $560.0 \mathrm{~K}$ & $291.3 \mathrm{~K}$ & $773.5 \mathrm{~kg} / \mathrm{m}^{3}$ \\
\hline Absolute deviation $(\%)$ & 0.1 & 0.5 & 0.2 \\
\hline
\end{tabular}

TABLE 8

Estimation of the boiling point, freezing point and liquid density of 2,3,3-trimethylpentane

\begin{tabular}{c|c|c|c}
\hline Groups & $\boldsymbol{T}_{\boldsymbol{b}}$ & $\boldsymbol{T}_{\boldsymbol{F}}$ & $\boldsymbol{d}_{\mathbf{2 0}}$ \\
\hline $5-\mathrm{CH}_{3}$ & $5 \times 33.6103$ & $5 \times 0.680429$ & $5 \times 0.653292$ \\
$1-\mathrm{CH}_{2}-$ & $1 \times 6.01945$ & $1 \times 0.123893$ & $1 \times 0.0467606$ \\
$1>\mathrm{CH}-$ & $1 \times(-22.8076)$ & $1 \times(-0.492776)$ & $1 \times(-0.57689)$ \\
$1>\mathrm{C}<$ & $1 \times(-51.0314)$ & $1 \times(-1.30057)$ & $1 \times(-1.20711)$ \\
\hline Correction terms & & & \\
\hline $2 \mathrm{C}\left(\mathrm{CH}_{3}\right)_{2}$ & $2 \times(-0.91393)$ & $2 \times 0.357568$ & $2 \times 0.0199574$ \\
$1 \mathrm{C}\left(\mathrm{CH}_{3}\right)$ & $1 \times(-0.55098)$ & $1 \times 0.264643$ & $1 \times 0.00470667$ \\
$\left.1 \mathrm{C}\left(\mathrm{CH}_{3}\right) \mathrm{C}_{(\mathrm{CH}}\right)_{2}$ & $1 \times 1.21475$ & $1 \times 0.0738494$ & $1 \times 1.4470 \mathrm{E}-04$ \\
$1 \mathrm{C}\left(\mathrm{CH}_{3}\right)_{2} \mathrm{C}\left(\mathrm{CH}_{3}\right)_{2}$ & $1 \times 2.12237$ & $1 \times 0.0990933$ & $1 \times(-0.00461323)$ \\
\hline Calculated value & $387.9 \mathrm{~K}$ & $156.8 \mathrm{~K}$ & $729.1 \mathrm{~kg} / \mathrm{m}^{3}$ \\
\hline Experimental value & $387.9 \mathrm{~K}$ & $172.5 \mathrm{~K}$ & $726.2 \mathrm{~kg} / \mathrm{m}^{3}$ \\
\hline Absolute deviation $(\%)$ & 0.0 & 9.1 & 0.4 \\
\hline \multicolumn{2}{c|}{}
\end{tabular}


TABLE 9

Estimation of the boiling point, freezing point and liquid density of 2-methyl-1-nonene

\begin{tabular}{c|c|c|c}
\hline Groups & $\boldsymbol{T}_{\boldsymbol{b}}$ & $\boldsymbol{T}_{\boldsymbol{F}}$ & $\boldsymbol{d}_{\mathbf{2 0}}$ \\
\hline $2 \mathrm{CH}_{3}$ & $2 \times 33.6103$ & $2 \times 0.680429$ & $2 \times 0.653292$ \\
$6-\mathrm{CH}_{2^{-}}$ & $6 \times 6.01945$ & $6 \times 0.123893$ & $6 \times 0.0467606$ \\
$1=\mathrm{CH}_{2}$ & $1 \times 32.9791$ & $1 \times 0.661299$ & $1 \times 0.652976$ \\
$1=\mathrm{C}<$ & $1 \times(-21.1492)$ & $1 \times(-0.660729)$ & $1 \times(-0.589958)$ \\
\hline Correction terms & & & \\
\hline $2 \mathrm{C}\left(\mathrm{CH}_{3}\right)$ & $2 \times(-0.55098)$ & $2 \times 0.264643$ & $2 \times 0.00470667$ \\
\hline Calculated value & $441.7 \mathrm{~K}$ & $210.2 \mathrm{~K}$ & $745.5 \mathrm{~kg} / \mathrm{m}^{3}$ \\
\hline Experimental value & $441.5 \mathrm{~K}$ & $208.9 \mathrm{~K}$ & $745.1 \mathrm{~kg} / \mathrm{m}^{3}$ \\
\hline Absolute deviation $(\%)$ & 0.05 & 0.6 & 0.05 \\
\hline
\end{tabular}

TABLE 10

Estimation of the boiling point, freezing point and liquid density of 1-dodecyne

\begin{tabular}{c|c|c|c}
\hline Groups & $\boldsymbol{T}_{\boldsymbol{b}}$ & $\boldsymbol{T}_{\boldsymbol{F}}$ & $\boldsymbol{d}_{\mathbf{2 0}}$ \\
\hline $1 \mathrm{CH}_{3}$ & $1 \times 33.6103$ & $1 \times 0.680429$ & $1 \times 0.653292$ \\
$9-\mathrm{CH}_{2^{-}}$ & $9 \times 6.01945$ & $9 \times 0.123893$ & $9 \times 0.0467606$ \\
$1 \equiv \mathrm{C}-$ & $1 \times 7.19358$ & $1 \times 0.0932368$ & $1 \times 0.0228269$ \\
$1 \equiv \mathrm{CH}$ & $1 \times 32.7471$ & $1 \times 0.615015$ & $1 \times 0.6395$ \\
\hline Calculated value & $490.5 \mathrm{~K}$ & $261.2 \mathrm{~K}$ & $773.5 \mathrm{~kg} / \mathrm{m}^{3}$ \\
\hline Experimental value & $488.0 \mathrm{~K}$ & $254.2 \mathrm{~K}$ & $780.9 \mathrm{~kg} / \mathrm{m}^{3}$ \\
\hline Absolute deviation $(\%)$ & 0.5 & 2.8 & 0.9 \\
\hline
\end{tabular}

TABLE 11

Estimation of the boiling point, freezing point and liquid density of 1-ethyl-cis-2-methylcyclopentane

\begin{tabular}{c|c|c|c}
\hline Groups & $\boldsymbol{T}_{\boldsymbol{b}}$ & $\boldsymbol{T}_{\boldsymbol{F}}$ & $\boldsymbol{d}_{\mathbf{2 0}}$ \\
\hline 2- $\mathrm{CH}_{3}$ & $2 \times 33.6103$ & $2 \times 0.680429$ & $2 \times 0.653292$ \\
4- $\mathrm{CH}_{2}-$ & $4 \times 6.01945$ & $4 \times 0.123893$ & $4 \times 0.0467606$ \\
2-CH< & $2 \times(-22.8076)$ & $2 \times(-0.492776)$ & $2 \times(-0.57689)$ \\
\hline Correction terms & & & \\
\hline Position cis- & -0.1424 & 0.0782603 & 0.00237399 \\
5 membered ring & 56.7392 & 1.63831 & 1.2041 \\
\hline Calculated value & $392.8 \mathrm{~K}$ & $171.0 \mathrm{~K}$ & $755.0 \mathrm{~kg} / \mathrm{m}^{3}$ \\
\hline Experimental value & $401.2 \mathrm{~K}$ & $167.2 \mathrm{~K}$ & $785.2 \mathrm{~kg} / \mathrm{m}^{3}$ \\
\hline Absolute deviation (\%) & 2.1 & 2.3 & 3.9 \\
\hline
\end{tabular}

TABLE 12

Estimation of the boiling point, freezing point and liquid density of 1,1-dicyclohexyl-dodecane

\begin{tabular}{c|c|c|c}
\hline Groups & $\boldsymbol{T}_{\boldsymbol{b}}$ & $\boldsymbol{T}_{\boldsymbol{F}}$ & $\boldsymbol{d}_{\mathbf{2 0}}$ \\
\hline $1-\mathrm{CH}_{3}$ & $1 \times 33.6103$ & $1 \times 0.680429$ & $1 \times 0.653292$ \\
$20-\mathrm{CH}_{2}-$ & $20 \times 6.01945$ & $20 \times 0.123893$ & $20 \times 0.0467606$ \\
$3>\mathrm{CH}-$ & $3 \times(-22.8076)$ & $3 \times(-0.492776)$ & $3 \times(-0.57689)$ \\
\hline Correction terms & & & \\
\hline 2 (6 membered ring) & $2 \times 57.9497$ & $2 \times 1.31182$ & $2 \times 1.18562$ \\
\hline Calculated value & $668.3 \mathrm{~K}$ & $304.1 \mathrm{~K}$ & $864.8 \mathrm{~kg} / \mathrm{m}^{3}$ \\
\hline Experimental value & $660.0 \mathrm{~K}$ & - & $875.4 \mathrm{~kg} / \mathrm{m}^{3}$ \\
\hline Absolute deviation $(\%)$ & 1.3 & - & 1.2 \\
\hline
\end{tabular}

TABLE 13

Estimation of the boiling point, freezing point and liquid density of 1,4-isopropyltoluene

\begin{tabular}{c|c|c|c}
\hline Groups & $\boldsymbol{T}_{\boldsymbol{b}}$ & $\boldsymbol{T}_{\boldsymbol{F}}$ & $\boldsymbol{d}_{\mathbf{2 0}}$ \\
\hline $3 \mathrm{CH}_{3}$ & $3 \times 33.6103$ & $3 \times 0.680429$ & $3 \times 0.653292$ \\
$1>\mathrm{CH}-$ & $1 \times(-22.8076)$ & $1 \times(-0.492776)$ & $1 \times(-0.57689)$ \\
$4=\mathrm{CH}-$ & $4 \times 6.33765$ & $4 \times 0.293538$ & $4 \times 0.0346579$ \\
$2=\mathrm{C}<$ & $2 \times(-21.1492)$ & $2 \times(-0.660729)$ & $2 \times(-0.589958)$ \\
\hline Correction terms & & & \\
\hline $\begin{array}{c}1 \text { (aromatic ring) } \\
1 \text { (substitution para-) }\end{array}$ & $1 \times 56.156$ & $1 \times 0.49712$ & $1 \times 1.20018$ \\
\hline Calculated value & $456.9 \mathrm{~K}$ & $241.7 \mathrm{~K}$ & $886.3 \mathrm{~kg} / \mathrm{m}^{3}$ \\
\hline Experimental value & $450.3 \mathrm{~K}$ & $205.2 \mathrm{~K}$ & $857.3 \mathrm{~kg} / \mathrm{m}^{3}$ \\
\hline Absolute deviation (\%) & 1.5 & 17.8 & 3.4 \\
\hline \multicolumn{4}{c}{}
\end{tabular}

TABLE 14

Estimation of the boiling point, freezing point and liquid density of 2,4'-dimethyldiphenylmethane

\begin{tabular}{c|c|c|c}
\hline Groups & $\boldsymbol{T}_{\boldsymbol{b}}$ & $\boldsymbol{T}_{\boldsymbol{F}}$ & $\boldsymbol{d}_{\mathbf{2 0}}$ \\
\hline $2 \mathrm{CH}_{3}$ & $2 \times 33.6103$ & $2 \times 0.680429$ & $2 \times 0.653292$ \\
$1 \mathrm{CH}_{2}$ & $1 \times 6.01945$ & $1 \times 0.123893$ & $1 \times 0.0467606$ \\
$8=\mathrm{CH}-$ & $8 \times 6.33765$ & $8 \times 0.293538$ & $8 \times 0.0346579$ \\
$4=\mathrm{C}<$ & $4 \times(-21.1492)$ & $4 \times(-0.660729)$ & $4 \times(-0.589958)$ \\
\hline Correction terms & & & \\
\hline $\begin{array}{c}\text { (aromatic ring) } \\
\text { (substitution ortho-) }\end{array}$ & $2 \times 56.156$ & $2 \times 0.49712$ & $2 \times 1.20018$ \\
1 (substitution para-) & $1 \times 0.85273$ & $1 \times 0.24589$ & $1 \times 0.0124819$ \\
\hline Calculated value & $564.0 \mathrm{~K}$ & $274.2 \mathrm{~K}$ & $989.0 \mathrm{~kg} / \mathrm{m}^{3}$ \\
\hline Experimental value & $571.0 \mathrm{~K}$ & - & $988.0 \mathrm{~kg} / \mathrm{m}^{3}$ \\
\hline Absolute deviation $(\%)$ & 1.2 & - & 0.1 \\
\hline
\end{tabular}

\section{CONCLUSION}

A new group-contribution method is developed to predict boiling point, freezing point and liquid density at $20^{\circ} \mathrm{C}$ of pure hydrocarbons. A number of structural groups were derived from the data bank, a particular care was taken in detecting differences between molecules, particularly in the case of isomers.

The results show that the proposed model for each property lead to good correlation with experimental data and give better deviations comparatively to other correlations recommended in literature. Besides, the proposed equations remain valid in the case of the heavy hydrocarbons, what is not the case of the other methods.

An extension of the present work will consist in the future in the study of other physical and thermodynamic properties such as critical and thermal properties with the same approach and the application of the established correlations to petroleum fractions. 


\section{REFERENCES}

Ambrose, D. (1980) National Physical Laboratory, Teddington, NPL Rep. Chem., 107.

Constantinou, L. and Gani, R. (1994) AICHE J., 40, 10, 16971710.

Constantinou, L., Gani, R. and O'Connell, J.P. (1995) Fluid Phase Equilibria, 103, 11-22.

Joback, K.G. (1984) S. M. Thesis in Chemical Engineering, Massachuset Institute of Technology, Cambridge, Mass.

Klincewicz, K. M. and Reid, R.C. (1984) AICHE J., 30, 1, 137.

Kreglewski, A. and Zwolinski, B. (1961) J. Phys. Chem., 65 , 1050 .
Lydersen, A.L. (1955) Coll. Eng. Univ. Wisconsin, Engineering Experimental Station Rept., 3.

Reid, R.C., Prausnitz, J.M. and Poling, B.E. (1987) The Properties of Gases and Liquids, 4th ed, McGraw-Hill, New York.

Riazi, M.R. and Al Sahhaf, T.A. (1995) Ind. Eng. Chem. Res., 34, $11,4145-4148$.

TRC Tables (1994) Thermodynamic Data Base, version 1.3.

Wuithier, P. (1972) Le pétrole: raffinage et génie chimique, tome 1, Éditions Technip, Paris. 